\title{
Applications of Queuing Theory in Quantitative Business Analysis
}

\author{
Dr. Daruri Venugopal, Professor, OPJS University, Churu, Rajasthan, India, \\ profdarurivg.edu@gmail.com
}

\begin{abstract}
Queuing Theory provides the system of applications in many sectors in life cycle. Queuing Structure and basic components determination is computed in queuing model simulation process. Distributions in Queuing Model can be extracted in quantitative analysis approach. Differences in Queuing Model Queue discipline, Single and Multiple service station with finite and infinite population is described in Quantitative analysis process. Basic expansions of probability density function, Expected waiting time in queue, Expected length of Queue, Expected size of system, probability of server being busy, and probability of system being empty conditions can be evaluated in this quantitative analysis approach. Probability of waiting ' $t$ ' minutes or more in queue and Expected number of customer served per busy period, Expected waiting time in System are also computed during the Analysis method. Single channel model with infinite population is used as most common case of queuing problems which involves the single channel or single server waiting line. Single Server model with finite population in test statistics provides the Relationships used in various applications like Expected time a customer spends in the system, Expected waiting time of a customer in the queue, Probability that there are $n$ customers in the system objective case, Expected number of customers in the system
\end{abstract}

Key Words : expected waiting time, life cycle, simulation process, single server, Queuing Structure, queuing problems.

\section{INTRODUCTION}

In Statistical Analysis queuing system consists of one or more servers that provide service of some sort to arriving customers. Queuing System identifies the customers arrival to find servers condition and customers generally join one or more queues in front of the servers, hence the name declared as queuing systems. We can identify several applications in life cycle, that can be described as queuing systems, such as bank-teller service, manufacturing systems, maintenance systems, computer systems, communications systems, hospital administration system, military organization system, educational curriculum system and many more. At analysis on a given queuing system involves study of its different operating characteristics like Queue length, System Length, Waiting Time in Queue, Total time in the System, Idle time of a server. It contains Input source as Population and different arrival patterns. Structure of a queuing system regulates the total analysis with respect to Calling population, Queuing process, Queue discipline and Service process-mechanism.

Classification along with Assumption. Probabilistic queuing models and Managerial Application of Queuing model applications always provides the major business decisions in Quantitate analysis. Solution of Queuing Models consists of two approaches like mathematical and simulation in this Analysis technic. Arriving people are called customers and the person providing the services is called a server. Serves may be in series or in a parallel form. Service time may be constant or variable and customers may be served singly or in batches.

\section{FEaTURes OF QUEUE SYSTEM}

We can determine several essential services as the major outputs features in Queue System.

$>$ Population - The input source is a set that contains the probable potential customers to come out for service related matters.

$>$ Limit of Queue - The limit of queue is sometimes restricted by server's functionality. If the limit is fixed by the server, the arrivals will be limited for a given time period. If there is no restriction then queue is said to be unlimited.

$>$ Queuing arrival pattern - The customers are expected to arrive at their own convenience and terms and conditions. The patterns of arrival times often like Poisson distribution, Exponential distribution and Enlarge distribution.

$>$ Under Queue Discipline priority Service rule the server gives priority to certain customers due to some importance or high cost group of the customers.

$>$ First in First out will provide the services as per the priority of order. We consider it as Queuing discipline in which the customers are served in the chronological order of their arrivals.

$>$ Last in First Out - It provides service in opposite order of arrivals of customers/orders, whoever comes last is served first and first obviously goes to last. 
$>$ Priority Service - Under this conditional service matters the server gives priority to certain customers due to some importance or high cost group of the customers.

$>$ Customer's Behavior - In daily routine service matters we can observe different attitudes of the customers with an intention to receive certain service may prepared to wait till he gets service. F he waits, hoping to get the service is called patient customer. For example Unsatisfied Customer, Dissatisfied Customer.

\section{COST ANALYSIS OF QUEUEING SYSTEM}

We can observe two conflicting costs in level of services like Cost of Offering Service and Cost incurred due to delay in offering service. If existing service level in terms of rate of service or number of service facilities is reduced the cost of operation comes lower. Similarly Increase in level of service increases the cost of operating service facilities. Waiting Costs analysis the reduction in existing service level will hike the customers waiting time or may result in long queues. Thus the optimum level of service can be obtained by achieving a balance between the above two costs or at the level where the total cost shows the minimum.

If existing service level in terms of rate of service or number of service facilities is reduced then the cost of operation always comes low. We consider Graph representation of Cost analysis of queuing system Cost in $\mathrm{Y}$ -axis ; Level service in $\hat{Y}$-axis, Total cost as central value, waiting cost which is parallel to $\hat{Y}$-axis. Service cost and minimum total cost optimum level of service are indicated as arc in graph part in cost analysis.

\section{BASIC STRUCTURE OF QUEUING SYSTEM}

We can identify the major parts of elements in Queuing System as Queuing process, Queue discipline, Service process and Input source.

Queuing Process - It refers the number of queues single, multiple or priority queues and their lengths. In Queuing process all the conditions in service system is unable to accommodate more than the required number of customers at a time. In such type of conditions no further customers are allowed to enter until more space is accommodated to new customers. Such type of queue depends on the layout of service mechanism and the length of a queue depends upon operational situations in Queuing process such as physical space, legal restrictions and attitude of the customers.

Queue Discipline - There are number of ways in which customers in the queue are served. Like static queue, dynamic, priority service, Emergency.

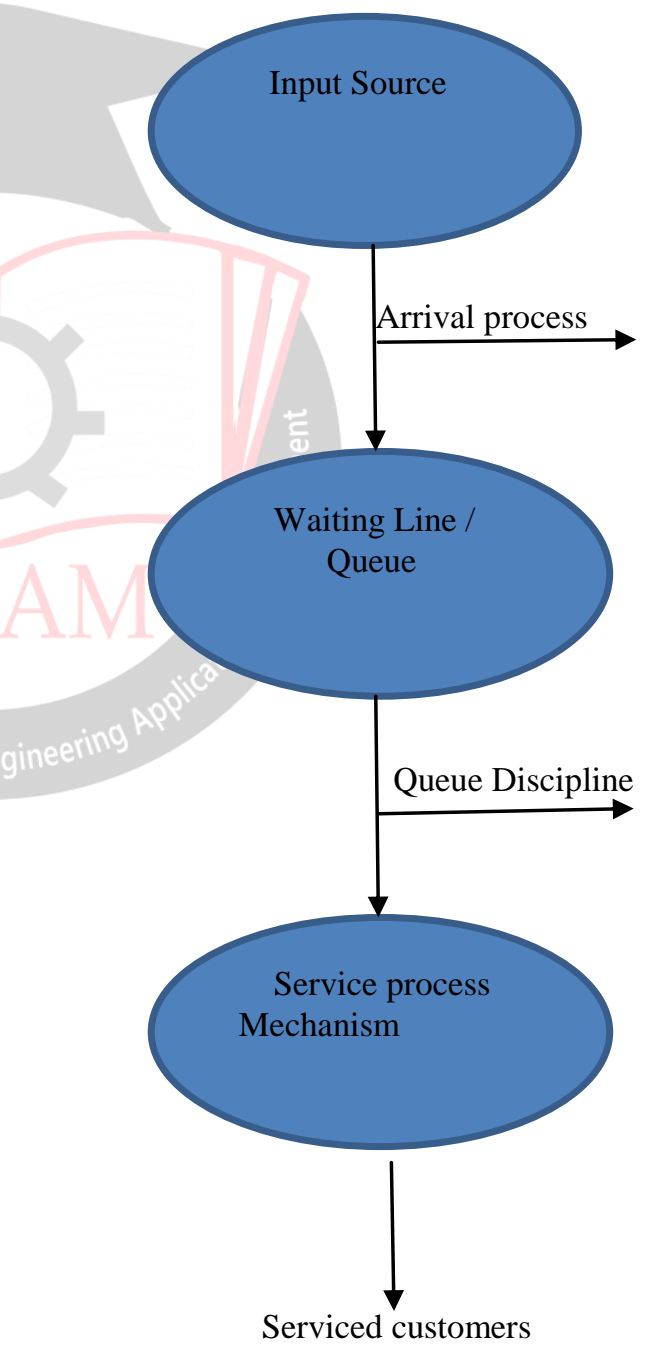

Fig .2 Queuing System Structure

Service process Mechanism - It concerned with the manner in which customers are serviced and leave the service system like Management Polices, Server's behavior, The distribution of service times, The arrangement of service facilities. 
Usually in Queuing System analysis service in random order conditions are selected for service at random irrespective of their arrivals in the queuing system.

It is mandatory that in all emergency cases an important customer is allowed to enter into the service immediately after entering into the system, even if a customer with lower priority is already in service. The interrupted service is resumed after the priority customer is served.

\section{QUEUING CHARACTERIZED OBJECTS}

We can classify major objects into Queue Discipline, Service Mechanism and Arrival Process models. All these Objects are like First in First Out and Last in first out process. It is observed that in systematic queuing system rules that a server uses to choose the next customer from the queue when the server completes the service of the current customer. In case of emergency Priority customers are served in order of their importance on the basis of their service requirements. In all mathematical assumptions, Statistical analysis the service mechanism of queuing system is specified by the number of servers each server having its own queue or a common queue and the probability distribution of customers service time.

It is very important that the arrival process which consists of describing how customers arrive to the system. If $\mathrm{T}_{\mathrm{i}}$ is the inter-arrival time between the arrivals of the [ $i-1]$ th order and same number of customers. We can determine the arrival frequency using mean and inter arrival time. Arrival may originate from one or several sources referred to as the calling population.

\section{QUEUING SIMPLIFICATION AND SINGLE CHANNEL PROCESS}

Basically we can assume two approaches like mathematical and simulation methods for solving queuing models. The mathematical approach has been applied where arrival and service time distributions are approximated by known mathematical distributions. We can consider the existing approaches like Binomial, Poisson, Exponential methods. If the cost associated with the performance measures is also known then optimal results can also be determined.

The simultaneous occurrence of arrivals and departures are also can be applied. The process helps in determining the probability distribution of the number of customers in the queuing system at a particular period of time. The probability distribution obtained is used to determine expected value of performance measures of queuing models.

The calling population is infinite
Queue discipline is first come first

$>$ In queuing discipline system Service times are described by an exponential distribution.

$>$ The average mean arrival rate is less than the mean service ratio

$>$ In Channel Queuing System the waiting space available for customers is infinite.

Waiting Line / Queue
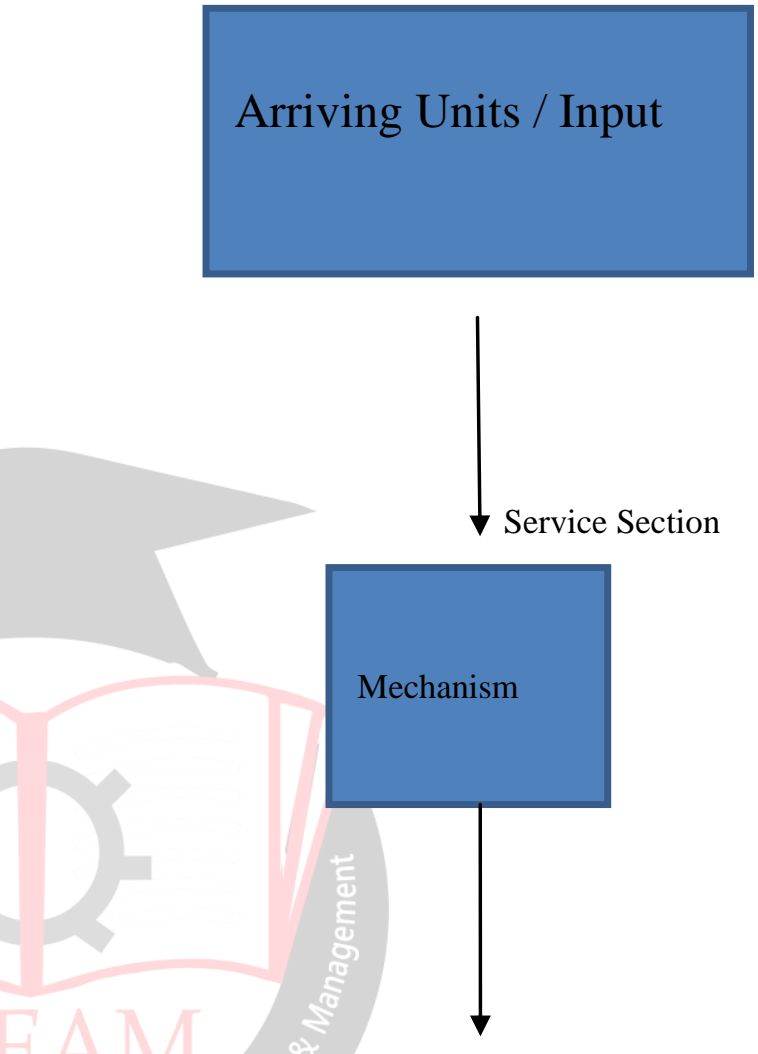

Output

Served Customers

Fig.3 Single Channel Queuing System

\section{QUEUING MODEL APPROACH}

Let us consider that the customer arrives at known intervals and the service time is known with certainty the queuing model shall be deterministic in all general conditions. If we assume customers come to a hospital cash counter every 5 minutes. Assuming that In this approach the interval between the arrival of any two successive customers is exactly 5 minutes.

Suppose further that the Hospital cash counter clerk takes exactly 5 minutes to serve a customers. Then the service rates are each equal to 12 customers per hour. In such case there will not be long queue. Similarly if he is supposed to attend for 15 customers cash dealings then he has to spend 4 minutes average to complete the task. In such case there will not be any queue.

In Queuing mechanism when a service rate is less than the arrival rate, the service facility cannot mix up with all the 
arrivals and eventually the system leads to an explosive situation. If we consider the mathematical approach for service rate computation where $\lambda$ is the arrival rate of customers per unit time and the service rate is $\mu$ per unit time then we can consider the following assumptions :

a) $\lambda>\mu \quad$; waiting line shall be formed which will increase indefinitely. Service system will fail.

b) $\lambda \leq \mu \quad$; waiting time shall not be there; service facility would be idle that is $[1-(\lambda / \mu)]$

c) $\mathrm{p}=\lambda / \mu$; ratio indicates the average utilization.

d) If $\mathrm{p}>1$, then system would ultimately fail.

e) If $\mathrm{p} \leq 1$ then system works and $\mathrm{p}$ will be busy.

\section{PROBABILISTIC QUEUING MODELS}

In Queuing models in several situations we can identify the method based on the assumption that one or more elements of the queuing system can be expressed only in probabilistic terms. We can declare and assume that all of the queuing models are of probabilistic type. If we consider mathematical condition that in Poisson exponential single server model-infinite population, finite population and Poisson exponential multiple server model infinite population.

We can consider Poisson exponential as indication of the customer arrivals and poison distribution which the service times are distributed exponentially. If the arrivals are considered as independent, with the average arrival rate equal to the parameter ' $\lambda$ ' per period of time, then according to the Poisson probability distribution, the probability that $\mathrm{n}$ customers will arrive in the system during a given interval $\mathrm{T}$, such that $\mathrm{T}=\mathrm{m} / \lambda$ with exponential value 'e'.

\section{CYCLIC APPLICATIONS}

$>$ Banks - Computing the optimum number of bank tellers for bank operations.

$>$ Theatres - Computing the number of ticket counters to reduce the queue of theatre goers waiting to buy tickets.

$>$ Telephone Exchange - Computing the optimum number to handle telephone calls.

$>$ Computers - Computing the requirement of CPU for handling the flow of computer programs through a computer system.

$>$ Supermarkets - Computing the number of counters for payment by the shoppers.

$>$ Airports - Computing the number of runways at airport terminals.

$>$ Industrial Units - Computing the number of machines required and the cost waiting and cost of serving are leading to least total cost so that the overall manufacturing cost reduces.
Structural Maintenance - Computing the number of repairmen required to repair the breakdown machines which arrive randomly. The optimum number of repairmen will make the sum of the cost of repairmen and cost of production loss from downtime, a minimum.

\section{BASIC MATHEMATICAL FORMS IN QUEUING MODELS}

a) Probability of server (when it is busy) $\mathrm{P}_{\mathrm{b}}=1-\mathrm{P}_{0}$

$=\lambda / \mu$

b) Probability of system with no customers waiting : $\mathrm{P}_{0}=1-\mathrm{P}_{\mathrm{b}} ;$ where $\lambda / \mu=\mathrm{P}_{\mathrm{b}}$

c) Probability of density function $\left(\mathrm{P}_{\mathrm{n}}\right)=\mathrm{P}^{\mathrm{n}}(1-\mathrm{p})$

d) Expected Size of $\operatorname{system}\left(\mathrm{L}_{\mathrm{s}}\right)=[\mu /(\mu-\lambda)]^{*} \mathrm{P}_{\mathrm{b}}$ $=[\mathrm{p} /(1-\mathrm{p})]$

e) Expected length of queue $\left(\mathrm{L}_{\mathrm{q}}\right)=\left[\mathrm{p}^{2} /(1-\mathrm{p})\right]$ $=\lambda^{2} /[\mu(\mu-\lambda)]$

\section{Example Problem-1 :}

The mean arrival rate to a service center is 1 per hour. The mean service time is 30 minutes. Assuming Poisson arrival rate and exponential servicing time then the process of evaluation is as below :

a) Mean service time calculation

b) Utilization factor calculation

c) Probability for two units in the system value

d) Expected time in minutes, customer has to wait in the system determination

Sol:- )

a) Mean service time : $(1 / \mu)=30$ mints. $\rightarrow$ $(30 / 60)=(1 / 2)$ hrs. $\rightarrow \mu=2$ per hour

b) Utilization factor : $(\lambda / \mu) \rightarrow(1 / 2)=0.5$

c) Probability for two units in the system : $\mathrm{P}(\mathrm{n}-2)$ $=(\lambda / \mu)^{2}=(0.5)^{2}=0.25$

d) Expected time in minutes, customer has to wait in the $\operatorname{system}(\mathrm{Ws})=[1 /(\mu-\lambda)]^{*} 60$

$60 /(2-1)=60$ minutes.

\section{Example Problem-2:}

In a distribution sequence decision making considering that receiving calls to a telephone booth as Poisson variant with an average duration of 30 minutes between one arrival and the next. During this process if duration of phone calls is assumed to be distributed exponentially, with a mean of 15 minutes.

Determine a) Computation of probability that a person has to wait at the booth 
b) Average length of non-empty queue

Sol:- )

a) Probability of a person that he has to wait at the booth :

$\mathrm{P}(\mathrm{n}>0) \quad 1-\mathrm{P}_{0}=\lambda / \mu \quad$ where

$\lambda=1 / 30=0.033$ person per minute and

$\mu=1 / 15=0.066$ person per minute

$\lambda / \mu=0.033 / 0.066=0.5$

b) Average length of non empty queue : $L=(\mu / \mu-\lambda)$

$=0.066 / 0.033=2$ customers

\section{CONCLUSION}

Queuing Analysis Applications provides the basic components of queuing model and distributions in queuing model. It determines the differences in queuing model with first come first service system, Queue Discipline concept provides the Single and Multiple service station with finite and infinite population. The average number of customers in the system those waiting to be and those being serviced will be identified. The average time that a customer spends in the system from entry in the queue to completion of service will also computed in analysis method. Under the rule Priority service the server gives priority to certain customers due to some importance emergency.

The efficiency and capacity of queue system can be increased by arranging the service systems in the effective way particularly when multi service facilities are available. Queuing process, Queue discipline, Service process mechanism are discussed for pure analysis of work flow. Queuing model Distributions are also classified for assumption simplifications.

\section{ACKNOWLEDGEMENT}

I am very much thankful to the Vice-Chancellor, Prof. Dr. Dalel Singh, Dean School of Science, Microbiology \& Agriculture, The Registrar of OPJS University. I wish to show my gratitude to my Academic Guru Dr. G. Manohar Reddy Garu and Dr. N. Goutham Rao Garu Management of Swamy Vivekananda Educational Trust, Hyderabad for their motivational thoughts and Support in Academics \& Research activities..

\section{REFERRENCES}

[1]. Stationary Marked Point Processes- An Intuitive Approach Chapman \& Hall, New York, Sigman,Karl(1995).

[2]. Stewart,W.J(1991),Numerical Solutions of Markov Chains. Marcl Dekker, New York.
[3]. Syski,R.(1986). Introduction to Congestion Theory in Telephones, $2^{\text {nd }}$ edn.Elsevier Ltd.

[4]. Takacs,Lajos(1962). Introduction to the Theory of Queues. Oxford University Press, New York.

[5]. . Research Paper on Sampling Theory and Execution of Sample Surveys in Statistical organizations, by Dr.D.Venugopal, IRJMETS; Vol-02,Issue-05,2020

[6]. Rubinstein, Reuven Y.(1986). Moknte Carlo Optimization,Simulation, and Sensitivity of Queueing Networks, Wiley,New York.

[7]. Saad Y.(1994),Numerical Methods for Large Eigenvalue Problems. Halstead Press, New York.

[8]. Saaty,Thomas L.(1961).Elements of Queueing Theory with Applications. McGraw Hill, New York.

[9]. Educational Evaluation and Analysis of Statistical Techniques - by Dr. Daruri Venugopal Research Paper UGC Journal, Vol.6, Issue:02, May-2020.

[10]. Stable Non-Gaussian Random Processes Samorodnitsky,Gennady and Taqqu, Murad S.(1994). Stochastic Models with Infinite Variance. Champan \& Hall, New York.

[11]. Statistical Applications of Confounding Techniques in Factorial Designs for Basic Science and Engineering Research paper by Dr.N.Ramya, Prof(Dr) Daruri Venugopal.

[12]. Stochastic Models in Operations Research, vol.I.McGrwa-Hill, New York Heyman.D.P. and Sobel M.J.(1982).

[13]. Hernandex-Lerma,Onesimo and Lasserre, Jean Bernard(1996).Discrete-Time Markov Control Processes: Basic Optimality Criteria.SpringerVerlag,New York.

[14]. Wagner, H.M., Principle of Operations Rosearch,Prentice-Hall of India,New Delhi,1975.

[15]. Trueman R.E. Quantitative Methods for Decision Making in Business Half-Saunders, New York,1981. 